\title{
LETTERS
}

Evidence-based medicine (EBM)

Paul Kamill

Toby Lipman

Antibiotic management of sore throat Suresh Pathak

Desmond Spence, David Baillie, David Byford and Clare Briggs

David M Lewis

Sensible drinking: were GPs influenced by the Government report?

Eileen FS Kaner, Catherine A Haighton and Brian R McAvoy
GP training in dermatology

EM Higgins, LC Fuller and

AWP du Vivier

Minor surgery in general practice OA Thurtle

Hormone replacement therapy Martin Rodgers and John Miller

Questionnaire response rates MJB Wilkinson

Spread of HIV from non-drug user to non-drug user

Rona Wyld, Roy Robertson and Jack Gilon

\section{Evidence based medicine}

Sir,

The article by Jacobson et al (July Journal $)^{1}$ seems to be a special pleading for a new form of evidence-based Medicine (EBM) for general practice, with different rules from the rest of medical practice. The article raises the issue of evidence being dependent on the context. In other words 'beauty is in the eye of the beholder'. Alternatively, evidence depends upon who is interpreting it. EBM sets standards ${ }^{2}$ which, if eroded, no longer represent evidence except in the context in which the rules are interpreted. Are we to have evidence-based practice that differs according to who is interpreting it? Does this also imply that general practice will be the poor relation in EBM?

Last year, Professor Black ${ }^{3}$ made an excellent case that EBM should not be reliant only on the randomized controlled trial (RCT). He argued that observational studies, regarded by some as having little value, might be just as valid as the RCT. There is a danger of falling into the trap of overinterpreting what evidence-based medicine is and is not. It is not prescriptive.

It is interesting how many articles have claimed the pedigree of EBM. At least this article admits that we have been practising EBM for many years. What the authors don't address is the reason why EBM has become the 'paradigm' to be signed up to.

Harold Sox is extremely persuasive when writing about probability. ${ }^{4} \mathrm{He}$ addresses several sticky issues that deal with the underlying philosophy of EBM. The quote that is appropriate here is, 'A profession is defined in part by whether it commits itself to setting standards for the services that society expects...One of the durable standards of the medical profession is providing the best possible care for patients...The new standard is to provide the best possible care at the lowest price'. This may be the 'bottom line'; however, we need to recognize that there have always been limits to what we could afford and there always will be..$^{5}$ A primary care-led National Health Service could be an even more expensive option if we relax the 'rules'.

None of this negates the premise that, as clearly stated in the article, the best external (and available) evidence should always be integrated with clinical expertise. Judgement is always likely to be called for in clinical decision making. ${ }^{6}$

\section{PAUL KAMILL}

Bradford Health Authority

New Mill

Victoria Road

Shipley

West Riding of Yorkshire

Bradford BD18 3LD

\section{References}

1. Jacobson LD, Edwards AG, Granier SK Butler CC. Evidence-based medicine and general practice. Br J Gen Pract 1997; 47: 449-452.

2. Evidence Based Medicine Working Group. Evidence-based medicine: a new approach to teaching the practice of medicine. JAMA 1992; 268: 2420-2425.

3. Black N. Why we need observational studies to evaluate the effectiveness of health care. BMJ 1996; 312: 1215-1218.

4. Sox HC. Probability theory and the interpretation of diagnostic tests. In: Sox HC (ed.) Common diagnostic tests. Use and interpretation. Philadelphia: American

5. Hampton JR. The end of clinical freedom. BMJ 1983; 287: 1237-1238.

6. McCormick J. The place of judgement in medicine. Br J Gen Pract 1994; 44: 50-51.

“C'est à l'Ètat qu'il appartient de déterminer, dans le domaine de la recherche, ce qui est le plus utile à l'intérêt publique et d'affecter à ces objectifs-là ce dont il dispose en fait de moyens et en fait d'hommes." (Charles de Gaulle, 1959)

Sir,

Jacobson et al (July Journal) advocate the 'appropriate use of the principles of evidence-based medicine' while warning that College of Physicians, 1990.
Note to authors of letters: Letters submitted for publication should not exceed 400 words. All letters are subject to editing and may be shortened. Letters may be sent either by post (please use double spacing and, if possible, include a Word for Windows or plain text version on an IBM PC-formatted disk), or by e-mail (addressed to journal@rcgp.org.uk; please include your postal address). All letters are acknowledged on receipt, but we regret that we cannot notify authors regarding publication.
'EBM could be over-emphasized [and] in general practice this would hinder further progress in developing its philosophy and practice. ${ }^{1}$ They warn of over-reliance on the biomedical model and of the danger of neglecting the personal and contextual perspectives in diagnosis.

If EBM was nothing more than the application of the principles of clinical epidemiology to biomedical problems, using only evidence from randomized controlled trials, the authors would be correct. However, they do not acknowledge the educational origins and purpose of contemporary EBM, which is 'a process of life-long, self-directed learning' in which the objective is principally to identify gaps in our knowledge when presented with patients' problems and then rectify them. ${ }^{2}$

My experience as a participant in two of the UK workshops in teaching evidence-based medicine, which have been held in Oxford, London, and Cardiff, suggests that the educational method (facilitated, small group, participant-directed learning) is more familiar in general practice and primary care than in many other disciplines. Moreover, we have been able to apply it to qualitative research. For example, Kai's work on parents' worries about acute illness in pre-school children ${ }^{3}$ was included in the teaching packs for the 6th UK workshop (Oxford, 6th-11th July, 1997), and my group used it successfully in one of our sessions.

At an earlier workshop, discussion of EBM's implications for primary care identified issues such as empowerment and the enhancement of our role as patients' advocates as likely consequences of practising EBM as general practitioners. ${ }^{4,5}$ If we carry it to its logical conclusion, we may find that it does change the culture of general practice, but in a beneficial way. The habit of forming structured, answerable questions and answering them with the best evidence available to us would change the consultation. If we are 
explicit about this process to patients and encourage them to share in the decision on how the evidence is used in their management it would alter the doctor-patient relationship. Patients would be empowered to make decisions on the basis of greater understanding of their problem rather than the doctor's authority.

It is also most important that, given the empowering nature of the EBM process, we do not assume that it is for doctors only, but enable the whole primary health care team to use it. For this reason, many prefer the term 'evidence-based practice'.

TOBY LIPMAN

\section{Collingwood Terrace \\ Jesmond \\ Newcastle upon Tyne}

\section{References}

1. Jacobson LD, Edwards AGK, Granier SK, Butler CC. Evidence-based medicine and general practice. Br J Gen Pract 1997; 47: 449-452.

2. Sackett DL, Richardson WS, Rosenberg W, Haynes RB. Evidence-based medicine: how to practice and teach EBM. London: Churchill Livingstone, 1997.

3. Kai J. What worries parents when their preschool children are acutely ill, and why: a qualitative study. BMJ 1996; 313: $983-$ 986.

4. Rogers S. Evidence-based learning for general practice. [Letter.] Br J Gen Pract 1997; 47: 52-53.

5. Lipman T, Rogers S, Jones Elwyn G Evidence-based medicine in primary care: some views from the 3rd UK workshop on teaching evidence-based medicine. Evidence-Based Medicine 1997 (in press).

\section{Antibiotic management of sore throat}

Sir,

I greatly welcomed the article 'A randomized controlled trial of antibiotics on symptom resolution in patients presenting to their practitioner with a sore throat' by Howe et al (May Journal). Although the authors concentrated more on the use of antibiotics, I particularly appreciated the introduction, where they pointed out that 'The prescribing behaviour of GPs is complex and the decision to prescribe an antibiotic for a sore throat is influenced by social considerations.'

It has been recorded that upper respiratory tract infections (UTIs) (including sore throat) account for nearly $25 \%$ of the cases in general practice. ${ }^{1}$ Unfortunately, there is little published material available on the subject.

I have just concluded a comparatively small study in my surgery for postgraduate studies, under the heading 'Upper res- piratory tract infections and their management in general practice.' I recorded the total number of consultations, and those concerning URTI, and how they were managed; e.g. was an antibiotic used or not? I undertook this study in my own surgery with three partners and a GP registrar, and compared my findings with two other practices within the same Family Health Services Authority area: one is a practice with two doctors operating from a health centre, and the other is a singlehanded practice in which the GP operates from a house converted into a surgery. The patients of both these practices are of a lower social class compared with my surgery, and the primary care services here are overused.

Antibiotics were more frequently prescribed by the practice that operates in the lower social class area. I requested that all the GPs indicate the reason for prescribing: on clinical grounds, to prevent secondary infections, patient going away on holiday, something had to be done, doctor did not know how to handle the consultation. From the data I received it seemed that prescriptions for antibiotics were issued on clinical grounds; however, did the doctor's vanity prevent him from saying anything else?

Another interesting feature that emerged was that the same practice freely prescribed items like decongestants, nose drops, and lintuses, which are readily available over the counter.

Little et al very rightly suggest that 'prescribing antibiotics enhances belief in antibiotics and intention to consult' and 'only marginally affects the resolution of symptoms'.2 But in the real world, one has to look beyond the throat. In my study I also requested information on the social and environmental conditions of those patients (or their parents) presenting with URTI. Analysis of the data disclosed the following significant factors: pollution (smoking, high-rise blocks of flats, overcrowding, no playing facilities for children, densely populated areas near to major roads), the absence of central heating (or the inability to afford it), singleparent families, and stressful family events. In general practice, an effective solution is usually the result of a holistic approach, which seeks to identify the patient's real reason for attending the surgery. In a nutshell, unless these social and environmental factors are addressed properly by the appropriate agencies, and psychological factors or stress-related symptoms are understood beyond the level of the throat, it is difficult to find an ideal solution for the management of URTI.

My own very small study also reveals that a prescription for antibiotics has very little or no effect on the management of URTI, but in general practice one has to resort to doing so (although it is a very bad attempt at a solution). Balint wrote (on the question of repeat prescriptions): 'The whole truth is that the peaceful repeat situation is only a facade behind which lie the remnants of hopeless struggle in which both parties were defeated.' I feel that this statement is equally appropriate in the context of issuing prescriptions for URTI or sore throats.

SURESH PATHAK
84 Parkway
Gidea Park
Romford
Essex RM2 5PL
Pathak@virgin.net

\section{References}

1. Lambert H. Morbidity in general practice. Utrecht: Huisartsenpers, 1984.

2. Little $\mathrm{P}$ et al. Open randomized trial of prescribing strategies in managing sore throat. BMJ 1997; 314: 722-727.

3. Balint $\mathrm{M}$ et al. Treatment of diagnosis: a study of repeat prescriptions in general practice. London: Tavistock Publications, 1970.

Sir,

The issue of sore throats was again raised in the paper by Howe et al (May Journal) in respect of the use of antibiotics, namely 'cefixime', to reduce the duration of symptoms. ${ }^{1}$

We feel very strongly that the readers of the $B J G P$ should recognize that antibiotics should not be used in the treatment of sore throat except under extreme circumstances. Antibiotics may perhaps shorten the duration of symptoms, ${ }^{2}$ but they also expose patients to possible side-effects. The prescribing of antibiotics feeds into doctor dependence, undermines patients self-reliance, and increases GP workload. ${ }^{2}$

The issue of Beta-Haemolytic Streptoccocal disease is often raised, but because only $5 \%$ of patients with sore throat consult their doctors, ${ }^{3}$ and no evidence exists to suppport antibiotic use as a public health measure, ${ }^{4}$ this can not be used as an argument.

In our opinion, the discussion should revolve around empowering patients to have the confidence to deal with minor illnesses and not perpetuate health neuroses that the health profession is responsible for.

DESMOND SPENCE DAVID BAILLIE DAVID BYFORD Clare Briggs 
Green Area

Maryhill Health Centre

41 Shawpark Street

Glasgow G20 9DR

\section{References}

1. Howe RW, Millar MM, Coast J, et al. A randomized controlled trial of antibiotics on symptom resolution in patients presenting to their general practitioner with a sore throat. Br J Gen Pract 1997; 47: 280-284.

2. Little P. Open randomized trial of prescribing strategies in managing sore throats. BMJ 1997; 314: 689-672.

3. Banks MH. Factors influencing demand for primary medical care in women aged 20-44 years: a preliminary report. Int J Epidemiol 1975; 4: 189-195.

4. Del Mar C. Managing sore throat: a literature review II. Do antibiotics confer benefit? Med J Australia 1992; 156: 644-649.

Sir,

I was interested to read about the antibiotic management of sore throat by Howe et al (May Journal). This randomized controlled trial comparing penicillin, cefixime, and placebo was flawed by small numbers and failure to follow up those patients lost to follow-up. I take issue with some of the points of the study:

There is probably no difference between antibiotic therapy and placebo if those patients lost to follow-up are assumed to have not responded

- There were six patients who failed to complete the course of antibiotics, yet they were included in the treatment group analysis

- The most important outcome measure is surely time taken to return to work; this was not formally evaluated.

I wonder if the authors of the study have performed a multivariate analysis with their data to analyse some of these confoundings?

DAVID M LEWIS

20 Cranbrook Drive

Maidenhead, Berkshire SL6 6SB

\section{Reference}

1. Howe RW, Millar MM, Coast J, et al. A randomized controlled trial of antibiotics on symptom resolution in patients presenting to their general practitioner with a sore throat. Br J Gen Pract 1997; 47: 280-284.

\section{Sensible drinking: were GPs influ- enced by the Government report?}

Sir,

Strang et al (Letters, April Journal) reported broad support from GPs for preexisting sensible drinking limits in a sur- vey carried out before the highly-publicized Government report on Sensible Drinking. ${ }^{1}$ This report recommended that up to four units of alcohol per day for men and up to three units per day for women would not accrue significant health risks. Given public familiarity with weekly drinking limits, ${ }^{2}$ the Sensible Drinking report was widely interpreted as advocating a new ceiling of 28 units per week for men and 21 for women. The response to this report by health experts was virtually unanimous in adhering to the established limits of 21 and 14 units per week respectively for men and women. But where did this conflicting information leave GPs who must advise the public?

We report one finding from a postal questionnaire survey of 411 randomly sampled GP principals from the Midlands, one per practice, concerning attitudes and practices towards preventive medicine and early alcohol intervention. One question asked GPs to identify the upper limit for alcohol consumption for healthy and nonpregnant women. By chance, the Sensible Drinking report was published in the middle of the survey period, providing an opportunity to study its influence on GPs. The response rate in this survey was $68 \%$ $(n=279)$, with $141(50.5 \%)$ GPs responding before the report was published and $138(49.5 \%)$ responding afterwards.

Of the 279 GPs in the survey, 224 $(80 \%)$ answered the question on alcohol limits. Average recommended limits from before and after publication of the report are shown in Table 1. Over the whole study period, the mean upper alcohol limit for men was 22.8 units $(\mathrm{SD}=5.8)$, although both the median and modal values were 21 , with $41 \%(n=89)$ of GPs reporting this limit. The mean upper alcohol limit for women was 15.6 units (SD = $4.5)$, although both the median and modal values were 14 , with $50 \%(n=108)$ of GPs reporting this limit.

There were no significant differences between GPs' reported limits for men and women before and after publication of the Sensible Drinking report. However, the proportion of GPs reporting 14 units for women fell from $57 \%$ to $42 \%$ over this period.

These results suggest that publication of the Sensible Drinking report did not significantly alter GPs' views on recommended limits of 21 and 14 units per week for men and women as endorsed by the three Royal Colleges. ${ }^{4}$ Because of the intense lay and media interest generated by the publication of the Sensible Drinking report, it is unlikely that GPs were unaware of the apparent upward shift in the Government's recommended limits. Thus, it is likely that GPs either chose to ignore the new recommendations or were reassured by the swift response by health experts in reaffirming the original limits. ${ }^{3}$

EILEEN F S KANER Catherine A Haighton BRIAN R MCAVOY

Department of Primary Health Care

The Medical School

Framlington Place

University of Newcastle

Newcastle upon Tyne NE2 4HH

Nick HEATHER

Centre for Alcohol and Drug Studies Newcastle City Health NHS Trust

Newcastle

\section{Acknowledgement}

This work was funded by the Yorkshire Regional Health Authority. Dr Kaner and Ms Haighton were supported by the Alcohol Education Research Council.

\section{References}

1. Inter-departmental Working Group. Sensible Drinking. The report of an interdepartmental working group. London: DOH, 1995.

Table 1. GPs' recommended upper limits for alcohol consumption (units/week) before and after publication of the Sensible Drinking report.

\begin{tabular}{lcc}
\hline & $\begin{array}{c}\text { Before report } \\
\mathrm{n}=141\end{array}$ & $\begin{array}{c}\text { After report } \\
\mathrm{n}=138\end{array}$ \\
\hline Limits for men & & \\
Mean (SD) & $22.5(5.4)$ & $23.1(6.3)$ \\
$95 \%$ confidence interval & $21.3-23.3$ & $22.1-24.5$ \\
Median & 21 & 21 \\
Mode & 21 & 21 \\
Limits for women & & $16.0(5.1)$ \\
Mean (SD) & $15.1(3.9)$ & $15.1-17.1$ \\
$95 \%$ confidence interval & $14.2-15.7$ & 14 \\
Median & 14 & 14 \\
Mode & 14 & \\
\hline
\end{tabular}


2. Medical Council on Alcoholism. Do not change the numbers, clarify the message. Alcohol and Alcoholism 1995; 30: 571575 .

3. Edwards G. Sensible drinking: doctors should stick with independent medical advice. BMJ 1996; 312: 1.

4. Royal College of Physicians, Psychiatrists, and General Practitioners. Alcohol and the heart in perspective - sensible limits reaffirmed. London: Royal Colleges, 1995.
D TOVEY

Department of General practice and Primary Care

King's College School of Medicine and Dentistry

Bessemer Road

London SE5 9JP

\section{Reference}

1. Poyner T. GP training in dermatology. [Letter.] Br J Gen Pract 1997; 47: 257.

\section{GP training in dermatology}

Sir,

We strongly support Dr Poyner's plea that dermatologists should play a key role in the training of GPs, ${ }^{1}$ and we are concerned about the recent trend to reduce or even omit dermatology from the curriculum.

The dermatologists at King's College Hospital have been proactive in ensuring that the diagnosis and management of skin disease retain a high profile throughout all levels of training. This begins at the undergraduate level, where enthusiastic, interactive teaching has fostered ambition in a significant number of graduates who go on to specialize or retain a special interest in dermatology. At the postgraduate level, regular seminars are held for GP trainees, and most trainees take the opportunity to sit in on clinics during their vocational training. For those in practice, 'dermatology masterclasses', clinical demonstrations, and study days are held. Finally, in conjunction with the local purchasing authority, we have recently pioneered an intensive three-month training programme of individual tuition for local GPs, with a view to honing clinical and minor surgical skills, thus widening the level of care across the community and optimizing the use of current limited resources. Initial feedback has been very positive and we await the final analysis upon completion of the scheme to assess the impact on referral patterns.

We concur that a knowledge of dermatology is essential for those working in general practice, and would hope that, through established links and a little enthusiasm on both sides, this goal is readily attainable.

\section{E M HigGINS \\ L C FULLER}

A W P DU VIVIER

Department of Dermatology

King's College Hospital

London SE5 9RS

\section{Minor surgery in general practice}

Sir,

I thought the brief statement that there were 'No complications' as a result of all the minor surgical activity reported by Brown and colleagues (April Journal) ${ }^{1}$ unlikely until I did an audit of the joint and soft tissue injections and aspirations I performed last year. One hundred and eleven procedures were done, of which 104 concerned patients whose notes were still available. When these notes were reviewed, looking particularly for immediate or later complications, only one patient experienced any problems. He had two complications, and it might be of interest to describe them as a warning to others.

The first complication occurred a few minutes after an aspiration of the patient's olecranon bursa when he was sitting in a chair after moving from the examination couch. He suddenly said he felt faint and then lost consciousness. He was moved onto the floor where he rapidly regained consciousness and left fully recovered after a few minutes. Three days later he returned with an apparently infected bursa, which responded to antibiotic therapy.

The suddeness of his syncope, occurring minutes after the procedure was over, took me by surprise, and I suspect that pressure on the bursa, resulting from moving him to the recovery postition, contributed to the infection.

Joint injections are a rewarding part of general practice, but, as with any other intervention, problems can arise quite unexpectedly from them.

O A THuRTLE

Woodbridge Road Surgery 165-167 Woodbridge Road Ipswich IP4 2PE

\section{Reference}

1. Brown JS, Smith RR, Cantor T, et al. General practitioners as providers of minor surgery - a success story. Br J Gen Pract 1997; 47: 205-210.

\section{Hormone replacement therapy}

Sir,

We reported in the March Journal that levels of serum oestradiol $\left(\mathrm{E}_{2}\right)$ in women receiving transdermal $\mathrm{E}_{2}$ therapy may be inadequate to protect bone. Diana Mansour makes a valid point when she speculates that the low median serum $\mathrm{E}_{2}$ level we observed might have been because most of our patients were using reservoir (37) rather than matrix (8) patches (July Journal). She quoted evidence suggesting the serum $\mathrm{E}_{2}$ levels attained with reservoir patches tend to be lower than with matrix formulations and subject to greater fluctuations.

Indeed, we did find the median $(95 \%$ confidence intervals $(\mathrm{CI})$ ) serum $\mathrm{E}_{2}$ was higher in matrix (204 pmol/L, 93 to 489) than reservoir (145 pmol/L, 126 to 172$)$ patch users even though the median $\mathrm{E}_{2}$ doses received in the two groups were similar $(0.79 \mu \mathrm{g} / \mathrm{kg} / 24 \mathrm{~h}$ and 0.81 $\mu \mathrm{g} / \mathrm{kg} / 24 \mathrm{~h}$ respectively). However, the 95\% CI was very wide for the matrix group due to the small numbers. Consequently, the differences in serum $\mathrm{E}_{2}$ levels between the matrix and reservoir users was not significant.

We feel the greatest factor contributing to the low serum $E_{2}$ level was simply that more than half the patients received an adequate $\mathrm{E}_{2}$ dose when the body weight and not just the dose delivery rate was taken into account. Thus, the median $\mathrm{E}_{2}$ dose $(95 \% \mathrm{CI})$ received by patients with serum $\mathrm{E}_{2}<150 \mathrm{pmol} / \mathrm{L}(0.71 \mu \mathrm{g} / \mathrm{kg} / 24 \mathrm{~h}$, 0.42 to 0.83 ) was significantly lower than those with serum $\mathrm{E}_{2} \geqslant 150 \mathrm{pmol} / \mathrm{L}(0.91$ $\mu \mathrm{g} / \mathrm{kg} / 24 \mathrm{~h}, 0.79$ to $1.03 ; P=0.003)$.

In answer to Diana Mansour's comments regarding theue of percutaneous $\mathrm{E}_{2}$ gels, we agree that these preparations may be of value in the prevention of postmenopausal bone loss. However, the rate of $E_{2}$ absorption is proportional to the surface area over which the gel is applied, which is hard to determine accurately and may vary from day to day. ${ }^{1}$ Therefore, if $\mathrm{E}_{2}$ gels were to be used, the serum $\mathrm{E}_{2}$ levels could not be relied on to assess the adequacy of replacement for bone protection, which was the pupose of our study.

MARTIN RODGERS 
Bridge House Medical Centre

JOHN MILLER

11 Ladybridge Road

Cheadle Hulme

Cheadle

Cheshire SK8 5LL

\section{Reference}

1. Sitruk-Ware R. Estrogen therapy during the menopause. Practical treatment and recommendations. Drugs 1990; 39: 203 217.

\section{Questionnaire response rates}

Sir,

Deehan et al (February Journal) $)^{1,2}$ make some interesting observations about the effect of cash inducements on postal questionnaire response rates and whether a low response matters. They identified and sent a questionnaire on four separate occasions to GPs and followed this up with a telephone call. They found that accessing and persuading the ultra-non-responding GPs to complete the telephone interview problematic.

Surely, a major factor affecting the response rate, not identified in their introduction, is the number of questionnaires received by GPs. In the same issue, there are 11 original articles, of which nine depend on questionnaires or interviews, six of which were directed towards GPs.

As a previous member of a local ethics committee, I received regular complaints from patients with rare conditions who were over studied and received neverending questionnaires. It may be that GPs are so special that they deserve special study, but perhaps a box could be included on the next questionnaire: 'Tick here if you do not with to be involved in further studies.'

\section{J B WILKINSON}

Department of General Practice

The University of Birmingham

The Medical School

Edgbaston

Birmingham

M.J.B.Wilkinson@bham.ac.uk

\section{References}

1. Deehan A, Templeton L, Taylor C, et al. The effect of cash and other financial inducements on the response rate of general practitioners in a national postal study. Br J Gen Pract 1997; 47: 87-90.

2. Templeton L, Deehan A, Taylor C, et al. Surveying general practitioners: does a low response rate matter? Br J Gen Pract 1997 47: $91-94$.

\section{Spread of HIV from non-drug user to non-drug user}

Sir,

Ten years ago extensive discussion took place about the likely course of the HIV epidemic in intravenous (IV) drug users in the United Kingdom (UK). Edinburgh's case study demonstrated something not seen elsewhere in the UK - epidemic spread. ${ }^{1}$ The same thing was happening in New York and some southern European companies, and, for those of us close to the debate, the major projected interest was the potential for spread from a 'high risk activity group' to a comparatively low risk activity group, namely the wider heterosexual community. ${ }^{2}$ Many observers felt that intravenous drug users being the epicentre of an epidemic might prove to burn out as an on-going problem rather than lead to the wider heterosexual community having only a comparatively small number of close sexual contacts. Interestingly, a significant number of the original drug-using group found it hard to believe that they had been infected by needle and syringe sharing, considering sexual risk to have been greater.

Ten years on from those discoveries, and 15 years since the virus spread so rapidly in the young Edinburgh drug users, our observations are of a smaller number of new seroconversions related to IV drug use, but with a steady number of new presentations of younger non-injecting drug users with heterosexually acquired HIV infections. ${ }^{3}$ The original cohorts of IV drug users now present a sorry clinical state of advanced disease, cahectic chaos, and miserable dementia. Last month, the first case of spread from non-drug user to non-drug user alerted us to the phenomenon that the experts said, back then, would indicate the presence of a real heterosexual epidemic completely removed from drug users - a young man with a dramatic and impressive seroconversion illness acquired from his female partner who had never used drugs but had been infected by HIV by her drug-using partner some years ago (the drug-using partner had died four years ago). Only the presence of such an unusually severe seroconversion illness drew our attention to the spread of the virus to this individual. Since then, two other cases have been identified where no seroconversion illness was noticed and a positive testing followed anxiety about exposure. A fourth case presented recently when a 34-yearold male was found to be positive for antiHIV when he donated blood. He denied risk factors but claimed that his female partner of several years' standing, who is also seropositive, was previously married to an intravenous drug user (IDU). She herself denies IDU or other risk activity. Three of these four cases have been female to male transmission, resulting presumably from the original larger male population infected during the early 1980s.

This is the real heterosexual epidemic, and is essentially removed from any 'high risk' activity other than heterosexual vaginal intercourse. Other examples of this have been suggested but unconfirmed in recent years when the presence of continued drug injecting could not be excluded. For us, however, these cases mark the epidemic moving on, this is reflected in our daily surgeries, which comprise many new, advancing cases of AIDS, which are increasingly in young (mainly female) non-drug users. Scottish figures show an increasing number of heterosexual transmissions: 57 in 1995 and 49 in 1996, but only three in 1996 had identified 'high risk' partners. ${ }^{3}$

RONA WYLD

Edinburgh Drug Addiction Study

1 Muirhouse Avenue

Edinburgh EH4 4PL

ROY ROBERTSON

Muirhouse Medical Group

1 Muirhouse Avenue

Edinburgh EH4 4PL

\section{JACK GILLON}

SE Scotland Blood Transfusion Service

Royal Infirmary of Edinburgh

1 Lauriston Place

Edinburgh EH3

\section{References}

1. Robertson JR, Bucknall ABV, Welsby PD, et al. Epidemic of AIDS-related virus (HTLV-III/LAV) infection among intravenous drug abusers. BMJ 1986; 292: 527 529.

2. Moss A. AIDS and intravenous drug use: the real heterosexual epidemic. BMJ 1987 ; 294: 389-390.

3. Scottish Centre for Information and Environmental Health (SCIEH). Weekly report. Scotland: SCIEH, 1997. 